\title{
Distribution and Antifungal Susceptibility ofCandida Species Isolated from Clinical Specimens at the University Teaching Hospital, Lusaka, Zambia
}

\author{
Kelvin L. Sarenje ${ }^{1}$, Chileshe Lukwesa-Musyani ${ }^{3}$, James C. L. Mwansa ${ }^{3}$, \\ Mulemba T. Samutela ${ }^{1}$, Annie Kalonda ${ }^{1}$, Trevor Kaile ${ }^{2}$, John Mwaba ${ }^{3}$, Geoffrey \\ Kwenda ${ }^{1}$ \\ ${ }^{I}$ Department of Biomedical Sciences, School of Medicine, University of Zambia, Lusaka, Zambia, ${ }^{2}$ Department \\ of Pathology and Microbiology, School of Medicine, Lusaka, Zambia, ${ }^{3}$ Department of Pathology and \\ Microbiology, University Teaching Hospital, Lusaka, Zambia
}

\begin{abstract}
Background:Candida species have emerged as successful pathogens worldwide and are associated with immunocompromised patients. Additionally, there is increasing resistance of Candida species to antifungal agents, and this has greatly contributed to the high morbidity and mortality amongst affected patients. In Zambia, little is known about the distribution of Candida species and their antifungal susceptibility patterns. Speciation of Candida species is important as knowledge of the infecting species is important for guiding therapy. The objective of this study was, therefore, to characterise Candida species isolated from different clinical specimens at the University Teaching Hospital in Lusaka.

Methods:This was a cross-sectional study involving the identification of 96 Candidaspecies from various clinical specimens, and determination of their antifungal susceptibility patterns. Identification of the isolates was achieved by the use of the API 20C AUX kit, followed by DNA sequencing of the Internal Transcribed Spacer region of the ribosomal DNA, whilst the agar-based E-test, using fluconazole, amphotericin B, flucytosine, and caspofungin, was used for antifungal susceptibility testing.

Results: Data obtained showed that Candida albicans were the predominant species (66.7\%), followed by $C$. lusitaniae (12.2\%), C. glabrata (6.7\%), C. tropicalis (5.6\%), C. parapsilosis (3.3\%), C. quilliermondii (3.3\%), C.pelliculosa (1.1\%) and C. keyr (1.1\%). Most of the Candida species exhibited high levels of resistance to fluconazole and amphotericin B, but were sensitive to caspofungin and flucytosine. C. albicans was resistant to fluconazole $\left(18.3 \%\right.$, ) with an $\mathrm{MIC}_{90}$ of $256 \mu \mathrm{g} / \mathrm{ml}$ and amphotericin B $(10 \%)$ with $\mathrm{MIC}_{90}$ of $1.5 \mu \mathrm{g} / \mathrm{ml}$. C. glabrata was the most resistant species against amphotericin B (66.6\%) with an $\mathrm{MIC}_{90}$ of $2 \mu \mathrm{g} / \mathrm{ml}$.C. albicans and most of the non-albicans species exhibited multi-drug resistance.
\end{abstract}

Conclusion: This study demonstrated that identification of Candida species to species level and susceptibility testing are important for accurate treatment of Candida infections.

Key Words: Candida species, Antifungal susceptibility, Multi-drug resistance, Zambia.

\section{INTRODUCTION}

Fungal infections caused by yeasts of the Candida genus are a major problem especially in immunocompromised patients or those hospitalised with underlying disease [1,2]. Candida species are frequent colonizers of the skin and mucous membranes of animals and their dissemination in nature is widespread $[3,4]$ There are over 350 heterogeneous Candida species but only a few have been implicated in human disease [5]. Although the majority of Candida infections are attributable to Candida albicans, there has been an increase in the rate of infections caused by non-albicans in various parts of the world [6,7,8]. Among these species $C$. glabrata has emerged as one of the most important opportunistic pathogens to infect a variety of human body sites [9,10], while C.parapsilosis often represents the second most commonly isolated Candida species from blood cultures in many parts of the world $[11,12]$. Other species are rarely encountered in clinical samples although there have been several reports describing infections caused by uncommon Candida species [13]. Identification of Candida species from clinical specimens has become increasingly important as a result of increasing numbers of immune-suppressed patients, increasing use of indwelling medical devices, immunosuppressive therapy and broad-spectrum antibiotics [9,14]. Many clinical laboratories, however, do not speciate the Candida species isolated from clinical samples unless specifically requested. Thus, local changes or trends in infection causing species are difficult to determine. In addition the intensive and long term use of antifungal drugs lead to a decline in susceptibility and resistance patterns of Candida species [15]. It should also be noted that Candida species differ in their antifungal susceptibility and virulence factors $[16,17]$ 
The objective of this study was to identify and determine the antifungal susceptibility patterns of Candida species isolated from various clinical specimens at the University Teaching Hospital, a tertiary referral hospital in Zambia.

\section{MATERIAL AND METHODS}

Study site

This was a laboratory-based cross-sectional study on 96Candida species isolated from 1456 clinical specimenthat included urine, sputum, high vaginal swab (HVS) and blood submitted to the Microbiology Laboratory from July, 2013 to September, 2014 at the University Teaching Hospital, Lusaka. Zambia.

\section{ISOLATION OF CANDIDA SPECIES}

Candida isolates were isolated from routine clinical specimens that included urine, sputum, HVS, and blood in the Microbiology Laboratory at University Teaching Hospital. The isolates were sub-cultured on Sabouraud s' Dextrose Agar (Mast Diagnostics, Merseyside, UK) labelled and stored in vials containing 50\% glycerol at $-70^{\circ} \mathrm{C}$ for further analysis.

Phenotypic Identification of Candida species.The isolates were identified by API 20 C AUX kit (bioMerieuxSA, 69280 Marcy I'Etoile, France). The test was performed and interpreted according to the manufacturer's protocol.

\section{GENOTYPIC IDENTIFICATION OF CANDIDA SPECIES \\ 1. Preparation of DNA Thermolysates}

Genomic DNA used in this study was primarily prepared by thermo lysis of fresh Candida cells. Briefly, a loopful of yeast cells from Sabourand Dextrose Agar (Mast Diagnostics, Merseyside, UK) were transferred into a micro centrifuge tube containing $400 \mu \mathrm{l}$ of $1 \mathrm{x}$ TE buffer (10mM Tris- $\mathrm{HCl}$ [pH 8.0], 0.1mM EDTA [pH 8.0]) and boiled at $100^{\circ} \mathrm{C}$ for $15 \mathrm{~min}$. After cooling, the DNA thermolysate was then stored at $-20^{\circ} \mathrm{C}$ until required.

\section{PCR Amplification of the Internal Transcribed Spacer Region}

The sequence were first amplified by PCR in a final volume of $50 \mu$ l containing $10 \mu l$ of the Candidathermolysate and $40 \mu \mathrm{l}$ PCR master mix: $200 \mu \mathrm{M}$ of each dNTP, $2 \mu \mathrm{M}$ of each primer, ITS1-F(5'TCCGTAGGTGAACCTGCGG-3') and ITS1-R (5'-GCTGCGTTCTTCATCGATGC-3'), 1x Mastermix (50mM $\mathrm{KCl}, 10 \mathrm{mM}$ Tris-HCl [pH 8.3], $1.5 \mathrm{mM} \mathrm{MgCl}_{2}, 0.001$ [wt/vol] gelatine, and 1.25U of Taqpolymerase [Fermentas Life Sciences, Glen Burnie, MD, USA]). The PCR reaction was set for $10 \mathrm{~min}$ at $95^{\circ} \mathrm{C}$ and then subjected to 35 cycles of amplification for 45 seconds at $95^{\circ} \mathrm{C}, 45$ seconds at $55^{\circ} \mathrm{C}, 1 \mathrm{~min}$ at $72^{\circ} \mathrm{C}$, followed by a 10 min extension step at $72^{\circ} \mathrm{C}$ on a Gene Amp 2700 PCR thermal cycler (Applied Biosystems, CA, USA). The presence of the ITS1 DNA sequence was detected by electrophoresis of $5 \mu 1$ of the PCR amplicons on a $1.5 \%$ SeaKem LE agarose gel (Lonza, Rockland, ME, USA) containing $0.5 \mu \mathrm{g} / \mathrm{ml}$ ethidium bromide. A 50bp DNA ladder (Thermo Scientific, Handover MD, USA) was used as a molecular weight standard, and all gels were visualised using the BiotopBiosens SC-645 Gel Documentation System (Biotech Co. Ltd, Shanghai, China).

\section{DNA Sequencing}

Prior to sequencing, the PCR products were first purified with the GeneJET PCR purification kit (Thermo Scientific, Hanover, MD, USA) by following the manufacturer's instructions. Forward and reverse linear amplification was performed in $10 \mu 1$ using $2 \mu 1$ of purified PCR product (about 20 to 200ng), $2 \mu 1$ BigDye Terminator v.3.1 Cycle Sequencing kit (Applied Biosystems Foster City, CA, USA), 1 $\mu$ l BigDye Sequencing Buffer (Applied Biosystems Foster City, CA, USA) and $1 \mu \mathrm{M}$ of ITS1-F or ITS1-R primer. Linear amplification consisted of 25 cycles of denaturation at $96^{\circ} \mathrm{C}$ for $10 \mathrm{~s}$, annealing at $60^{\circ} \mathrm{C}$ for $30 \mathrm{~s}$ and elongation at $72^{\circ} \mathrm{C}$ for $60 \mathrm{~s}$ using Gene Amp 2700 PCR thermal cycler (Applied Biosystems, CA, USA). Fluorescence-labelled DNA was purified using the ethanol precipitation method (Ausubelet al., 2001). Briefly, the entire extension were transferred into $80 \mu \mathrm{l}$ of freshly prepared precipitation solution $(3 \mu \mathrm{l}$ of $3 \mathrm{M}$ sodium acetate [pH 4.6 ], 62.5 $\mu 1$ of non-denatured $95 \%$ ethanol and $14.5 \mu \mathrm{l}$ deionised water), incubated for at least $1 \mathrm{hr}$ at room temperature and centrifuged at $14000 \mathrm{rpm}$ for $20 \mathrm{~min}$. After carefully removing the supernatant, $250 \mu 1$ of $70 \%$ ethanol was added to the pellet, vortexed and the contents re-centrifuged at $14000 \mathrm{rpm}$ for $8 \mathrm{~min}$. The ethanol was carefully aspirated and the pellet air-dried for 15 mins at room temperature. The samples were analysed at the Inqaba Biotechnical Industries Sequencing Facility (Pretoria, South Africa) on an ABI PRISM 3730XL DNA analyser (Applied Biosystems, Foster City, CA, USA). The sequence reads were edited using RidomTraceEdit Software (Ridom Bioinformatics GmbH, Würzburg, Germany). The obtained sequences were compared with those available in GenBank by BLAST searches. Sequences that showed at least $98 \%$ sequence identity when compared to those in GenBank were preliminary considered as identified species [18]. 


\section{Antifungal Susceptibility Testing}

The antifungal susceptibility testing of isolates in this study was performed by the agar-based Epsilometer test (E-test) method. RPMI-1640 agar (Remel, Lenexa, USA) supplemented with $2 \%$ glucose and morpholinepropanesulphonic acid buffer (MOPS) (Remel, Lenexa, USA) in 130mm diameter plates was used. The plates were inoculated by dipping a sterile swab into a yeast cell suspension adjusted to $0.5 \mathrm{McF}$ arland standard units $\left(10^{6} \mathrm{cells} / \mathrm{ml}\right)$ using a turbidometer (Oxoid Integrated Technologies Ltd, England). The standardised inoculum was then streaked across the surface of the agar in four directions. The plates were dried at ambient temperature for 15 minutes before applying the E-test strips. The Candida isolates were tested against the following antifungal agents: 0.016-256 $\mathrm{\mu g} / \mathrm{ml}$ fluconazole (LiofilChem Diagnostic Ltd, Italy), 0.002$32 \mu \mathrm{g} / \mathrm{ml}$ flucytosine (LiofilChem Diagnostic Ltd, Italy), 0.002-32 $\mu \mathrm{g} / \mathrm{ml}$ amphotericin B (LiofilChem Diagnostic Ltd, Italy) and $0.02-32 \mu \mathrm{g} / \mathrm{ml}$ for caspofungin (LiofilChem Diagnostic Ltd, Italy). These antifungal agents are used for the treatment of Candida infections in Zambia. The minimum inhibition concentrations (MIC) endpoints were determined after 24 and 48 hours of incubation at $35^{\circ} \mathrm{C}$. The resistance breakpoints for antifungal agents were as follows: fluconazole $\geq 64 \mu \mathrm{g} / \mathrm{ml}$, amphotericin B $\geq 1.0 \mu \mathrm{g} / \mathrm{ml}$, flucytosine $\geq 32 \mu \mathrm{g} / \mathrm{ml}$, and caspofungin $\geq 32 \mu \mathrm{g} / \mathrm{ml}[19,20]$

\section{DATA ANALYSIS}

The data was analysed using the Strata software version 12.1 (Strata, California, USA). Categorical variables were compared using t-test and this included susceptibility to fluconazole and amphotericin $\mathrm{B}$. The tests were interpreted at 5\% significance level (two-sided) and 95\% Confidence Interval. A p-value of $<0.05$ was taken as indication of statistical significance.

\section{ETHICS CONSIDERATIONS}

Ethics approval for this study was granted by University of Zambia Biomedical and Research Ethics Committee. The ethics clearance certificate Number 004-05-14.

\section{RESULTS}

Out of the 96 culture isolates, 93.7\% (90/96) were identified as Candida species, while 6.3\% (6/96) were non-Candida species. These organisms were isolated from urine, sputum, HVS and blood. The predominant species was $C$. albicans $(66.7 \%, 60 / 90)$, followed by $C$. lusitaniae $(12.2 \%, 11 / 90), C$. glabrata (6.7\%, 6/90), C. tropicalis $(5.6 \%, 5 / 90)$, C. parapsilosis $(3.3 \%, 3 / 90)$, C. guilliermondii $(3.3 \%, 3 / 90)$ C. pelliculosa $(1.1 \%, 1 / 90)$ and $C$. kefyr $(1.1 \%, 1 / 90)$ (Figure 1).Urine was the most common source of Candida species, accounting for $41.1 \%$ (37/90) of the isolates, followed by sputum $(23.3 \%, 21 / 90)$, HVS $(22.2 \%, 20 / 90)$ and blood (13.3\%, 12/90) (Table 1).To confirm the identity of the Candida species identified in the fore-going section, PCR and DNA sequencing of the ITS1genomic region of the isolates was carried out (Figure 2). This also included the identification of the 6 unidentified species isolated from the different specimens. Using DNA sequencing it was noted that two isolates of $C$. albicans were misidentified as either C. famata or C. krusei, while one isolate of $C$. tropicalis was misidentified as $C$. parapsilosis by phenotypic method (Table 2). The six unidentified species were identified as being Pichia kudrianvzevii isolated from urine, Cryptococcus neoformans isolated from blood, Saccharomyces cerevisiae from blood, Trichosporumjaponicum from urine, and two species of Cyberlindnerafabianii from blood (Table 3).

C. albicans was resistant to fluconazole $(18.3 \%, 11 / 60)$, amphotericin B $(10 \%, 6 / 60)$, flucytosine $(8.3 \%, 5 / 60)$ and caspofungin $(3.3 \%, 2 / 60)$. C.lusitaniae showed resistance to fluconazole $(22.2 \%)$, and amphotericin B (22.2\%). Both C. glabrata and C. tropicalis strains were resistant to amphotericin B by $66.6 \%$ and $30 \%$, respectively. C. parapsilosis was only resistant to flucytosine (33.3\%), while C.quilliermondiiwas resistant to fluconazole, amphotericin B, flucytosine and caspofungin by $33.3 \%$. C. pelliculosa and C. kefyrstrains were sensitive to all the antifungal agents tested (Table 3). The lowest $\mathrm{MIC}_{50}$ observed for flucytosine was $0.004 \mu \mathrm{g} / \mathrm{ml}$ for $C$. lusitaniae and C. glabrata strains. The highest $\mathrm{MIC}_{50}$ observed for fluconazole was $256 \mu \mathrm{g} / \mathrm{ml}$ for $C$. quilliermondii. The $\mathrm{MIC}_{50}$ for $C$. albicans, the most isolated species, were $2 \mu \mathrm{g} / \mathrm{ml}$ for fluconazole, $0.75 \mu \mathrm{g} / \mathrm{ml}$ for amphotericin B, $0.016 \mu \mathrm{g} / \mathrm{ml}$ for flucytosine, and $0.25 \mu \mathrm{g} / \mathrm{ml}$ for caspofungin. The lowest $\mathrm{MIC}_{90}$ observed for flucytosine was $0.004 \mu \mathrm{g} / \mathrm{ml}$ against $C$. glabrata, and the highest $\mathrm{MIC}_{90}$ observed for fluconazole was $256 \mu \mathrm{g} / \mathrm{ml}$ against C. albicans, C. tropicalis and C. quilliermondii. The $\mathrm{MIC}_{90}$ for fluconazole against $C$. lusitaniae and C. glabrata were $96 \mu \mathrm{g} / \mathrm{ml}$ and $16 \mu \mathrm{g} / \mathrm{ml}$, respectively (Table 3). Most of the drug resistant Candida species (79.3\%, 23/29) were from HIV- positive than from HIV-negative (20.7\%) patients (Figure 2).C. albicans was the most drug-resistant strain in both HIV-positive $(56.5 \%$ ) and HIV-negative(50\%) patients (Figure 3).The Mean MICs of antifungal agents on Candida species from HIVpositive and HIV-negative participants were 52.6 and 27.9, respectively, for fluconazole $(p=0.17)$. Those for amphotericin B were 1.66 for HIV-positive and 2.39 for HIV-negative individuals $(p=0.51)$, while those for 
flucytosine were 3.73 for HIV-positive and 1.58 for HIV-negative individuals $(p=0.24)$. The Mean MICs for caspofungin was 2.11 for HIV-positive and 1.97 for HIV-negative individuals ( $p=0.92$ ) (Table 4).

Multi-drug resistance to a combination of four and two antifungal agents was observed in 5 Candida isolates. Multi-drug resistant was defined as isolates being resistant to more than two classes of antifungal agents. $C$. albicans showed 40\% MDR pattern to fluconazole, amphotericin B, flucytocine and caspofungin, while, $C$. lusitaniae and C. quilliermondii showed 20\% MDR pattern to all antifungal agents tested (Table 5).

\section{DISCUSSION}

In recent years, Candida infections in hospitalised patients have been on the increase [21,22]. This increase has been associated with surgical interventions, intensive care treatment, extreme of age, metabolic disorders, neutrophil dysfunction, and immunodeficiency states among others [23]. This calls for accurate identification of Candida species to select the most effective therapeutic strategies to control invasive fungal infections [24,25]. Data presented in this study demonstrate the occurrence of a variety of culturable fungal species obtained from different clinical specimens, which were mainly Candida species and a few non-Candida species. Among the Candida species, the predominant isolate was $C$. albicans, followed by $C$. lusitaniae, $C$. glabrata, C. tropicalis, C. parapsilosis, C. guilliermondii, C. pelliculosa and C. keyr. The non-Candida species included Pichia kudrianvzevii, Cryptococcus neoformans, Saccharomyces cerevisiae, Trichosporumjaponicum and Cyberlindnerafabianii. An important observation in our study is that the commonly isolated Candida species was $C$. albicans, and this corroborates with findings in other studies worldwide [26,27,28]. The predominance of this species may be attributed to virulence, conferring upon its enhanced capacity for colonisation and pathogenic activity for humans [29,30].C. albicans is the predominant species regardless of the immune status of the patient [31,32]. Another significant finding was that a number of non-albicans species were also isolated which shows their importance as pathogens in clinical specimens. This was consistent with Tanzanian and Iranian studies which also showed that $C$. albicans was a dominant species, while non-albicans Candida species were present in lower proportions [21,33].Phenotypic methods employed in this study misidentified three Candida isolates, and failed to identify the non-Candida fungal isolates: Pichia kudrianvzevii, Cryptococcus neoformans, Saccharomyces cerevisiae, Trichosporumjaponicum and Cyberlindnerafabianii. However, with the aid of molecular methods using the ITS region, the isolates were correctly identified.

The antifungal susceptibility testing is an important tool in the management of Candida infection because it promotes accurate administration of antifungal agents, and as an aid in drug development as well as a means of tracking the development of antifungal resistance in epidemiologic studies $[34,35,36]$. High resistance to fluconazole and amphotericin B, with high MIC levels, was observed with C. albicans, C. lusitaniae and $C$. glabrata, which were the most, isolated species in this study. There was a significant difference in the resistance pattern of $C$. albicans between fluconazole and amphotericin B $(p=0.001)$. Similar observations were made in an Iranian study in which $C$. albicans isolates were resistant to fluconazole, and usually have a poor response to treatment [37].The isolates of $C$. glabrata were the most resistant of all the Candida species against amphotericin B. This was consistent with a South African study which showed C. glabrata with high resistance to amphotericin B, and typically exhibited reduced susceptibility to amphotericin B [38].

The isolates of $C$. guilliermondii and $C$. parapsilosis showed relatively higher caspofungin MICs compared to other Candida species in this study. This was in keeping with studies conducted in South Africa and Tanzania where high caspofungin MICs were reported against C. guilliermondii and C. parapsilosis $[33,39]$.Comparing the Mean MIC values in this study, Candida isolates from HIV-positive patients showed higher values of resistance than isolates from HIV- negative patients. These results corroborate with findings in South African studies where drug resistance was reportedly high in HIV-positive than HIV-negative patients [40].The MDR observed in five isolates of Candida isolates, with $C$. albicans, being the predominant resistant, mostly to fluconazole and amphotericin B. In fact all the three species of $C$. albicans that showed an MDR pattern were isolated from HIV-positive patients. These findings on MDR pose serious clinical challenges as they seem to suggest the emergence of MDR Candida strains in hospitalised patients at the University Teaching Hospital in Lusaka.

\section{CONCLUSION}

This study demonstrated that C.albicans was the predominant Candida species causing infection at the University Teaching Hospital, and corroborates findings from other parts of the world. C. lusitaniae and $C$. glabrata were the most common non-albicans species isolated. Most of the organisms were isolated from HIVpositive patients, and were the most drug resistant. C. albicans, C.lusitaniae and C. glabrata were the most resistant species to all the antifungal agents tested. Caspofungin was the most effective drug compared to fluconazole and amphotericin B, two of the most widely used antifungal agents at the University Teaching Hospital. This study was the first to characterise Candida species using both phenotypic and molecular methods, and determine the antifungal susceptibility patterns in Zambia. 


\section{COMPETING INTERESTS}

The author declare that they have no competing interests

\section{Authors' contributions}

KLS, CLS and GK conceived and designed the study, analysed the data, and drafted the manuscript. MTS, AK and JM performed microbiological works. JCLM and TK helped study design and draft of the manuscript. All authors reviewed and approved the final manuscript.

\section{ACKNOWLEDGEMENTS}

This work was supported by the Ministry of Health, Department of Biomedical Sciences, School of Medicine, Department of Pathology and Microbiology, University Teaching Hospital of Zambia and Prof. Paul Kelly of TROPGAN, Lusaka, Zambia.

\section{REFERENCES}

[1] Van de Veerdonk, F. L., Netea, M. G., Jooten, W. M. and Kullberg. Novel strategies for the prevention and treatment of Candida infection. Federation of European Microbiologicalsocieties.2010;34: 10631075.

[2] Kumamoto, C. A., Bruzual, I. and Pierce, J. C.Immunosensing during colonisation by Candida albicans. Journal of Trends in Microbiology.2011;19: 263-267.

[3] Ruhnke, M., Paiva, J., Grigos, I., Miller, P., Marcek, T. and Kantechi, M. (2011).Anidulation for candidaemia in non-neutropenic intensive care unit patients.Journal of Critical Care, 15: 240.

[4] Udayalaxmi, Shani, J. and Diney, D. Comparison between virulence factors of Candidaalbicans and nonalbicans species of Candida isolated from genitourinary tract. Journal of Clinical and Diagnostic Research.2014;8: 15-17.

[5] Williams, D. W., Kuriyama, T., Silva, S. and Micheal, A. Candida biofilm and oral candidiasis: treatment and prevention. Journal of Periodontology.2011;55: 250-265.

[6] Rogers, T. R. Antifungal drug resistance: limited data, dramatic impact. International Journal of Antimicrobial agents.2006; 27: 7-11.

[7] Nishikaku, A. S., Melo, A. S. and Colombo, A. L. Geographic trends in invasive candidiasis, Journal of current fungal infections. 2010;4: 210-218.

[8] Howard, S. J., Livermore, J., Sharp, A. and Hope, W. W. Pharmacodynamics of echinocandinsagainistCandida glabrata in neutropenia hosts.Journal of Antimicrobial Agents and Chemotherapy.2011; 55: 4880-4887.

[9] Gizachew, Y., Daniel, A., Yimtubezinash, W. and Chandrashekhar, U. Prevalence of candiduria in diabetic patients attending Gondar University Hospital.Iranian Journal of Kidney Disease.2013;7: 102106.

[10] Zahra, S., Maryam, A., Zahra, S., Ali, Z. M. and Ahmed, S.Candiduria in children and susceptibility patterns of recovered Candida species to antifungal drugs in Ahvaz. Journal of Nephropathology.2014;2: 122-128.

[11] Delfino, D., Scordino, F., David, A. and Romeo, O. Potential association of specific Candida parapsilosis genotypes, blood stream infection and colonisation of health worker's hands. (2014). Journal of Clinical Microbiology and Infection.2014;20: 46-51.

[12] Quindos, G. Epidemiology of candidaemia and invasive candidiasis. Journal of Clinical Microbiology and Infection.2014; 20: 690-691.

[13] Brandt, M. E. and Lockhart, S. R.Recent taxonomic developments with Candida and other opportunistic yeast.Journal of fungal infection.2012;6: 170-177.

[14] Chaitanya, T., Vandam, K., Ruqaiyah, J. and Chiranjay, M. Antifungal susceptibility patterns, in-vitro production of virulent factors, and evaluation of diagnostic modalities for the speciation of pathogenic candida from blood stream infections and vulvovaginal candidiasis. Journal of Pathogens. 2014;2014: 8.

[15] Shakiba, E., Davarpanah, M. A. and Badiee, P. Distribution of antifungal susceptibility of Candida species from mucosal sites in HIV-positive patients.Journal of Iranian Medicine.2011; 13: 282-287.

[16] Chander, J., Singla, N., Sidhu, S. K. and Gombars, S. Epidemiology of Candida blood infections. Journal of Infections in Developing Countries.2009; 16: 670-675.

[17] Ibrahim, B., Roy, A. K., Hounda, H. and sima, T. Identification, Typing, Antifungal Resistance and Biofilm Formation of Candida isolates from Lebanese Hospital Patients. Journal of Biomedical Research International.2014;10: 1155 - 2014.

[18] Sulaiman, S. P., Singh, R. and Mandal, J. Fungal profile of funguria cases at a tertiary care hospital in southern India.Indian Journal of Research.2014;140: 556-559. 
[19] EUCAST. Breakpoint tables for interpretation on minimum inhibition concentration and zone diameters. 2012; version 2.0

[20] CLSI.Performance Standards for Antimicrobial Susceptibility Testing.CLSI Document M100-S24.2014; Wayne, PA, USA.

[21] Namkinga, L. A. Diversity of pathogenic Candida species colonising women with and without vaginitis. Journal of Biology and Life Science.2013;4: 153-170.

[22] Daiichi, M., Masafumi, S., Jose, B., Junzo, S. and Kazunori, T. Distribution of Candida species isolated from blood culture in hospitals in Osaka.Journal of Infectious Disease and Chemotherapy.2014; 20: 558562.

[23] Clarissa. O., Theresa, L., Paul, T., Mike, S. and Shamez, L. The epidemiology of neonatal and paediatric candidemia in England and Wales.Journal of Paediatric Infectious Disease.2013; 32: 23-26.

[24] Ali, A. L. (2011). Molecular typing and in vitro fluconazole susceptibility of Candida species isolated from diabetic and non-diabetic women with vulvovaginal candidiasis in India.Journal of Microbiology, Immunology and Infection.2011;44: 166-171.

[25] Annette, W. F., Deanna, A. S., Dora, I. M. and Nathan, P. W. Impact of new antifungal breakpoints on antifungal resistance in Candidaspecies.Journal of Clinical Microbiology.2014;52: 999-997.

[26] Jia, X. M., Ma, Z. P., Gao, P. H., Zhang, J. D. and Wang, Y. (2008). RTAZ, a novel gene involved in azole resistance in Candida albicans. Journal of Biochemistry and Biophysical research communications.2008; 373: 631-636.

[27] Schoch, C. L., Seifert, K. A., Huhndorf, S., Robert, V. and Crous, P. W. Nuclear ribosomal internal transcribed spacer region as a universal DNA barcode marker for fungi.Journal of BioScience.2012; 39: 4042- 4051

[28] Bartelli, F.T., Ferreira, R. C., Colombo, A. L and Briones M. R.Intraspecific of comparative genomics of Candida albicansmitochondria.Journal of Infection, Genetics and Evolution.2013;14: 302-312.

[29] Alice, G. S., Clemens, J. H., Stanley B. and Frans, M. K. Beyond the wall: Candida secretes to survive. Federation of European Microbiological societies.2012;10: 12-17.

[30] Ostrosky- Zeichner L., Shoham S. and Vazquez J.A randomised double - blind, placebo-controlled trial of Casponfugin prophylaxis followed by therapy for invasive candidiasis in high -risk adults in critical care settings. Journal of Clinical Microbiology and Infectious Disease.2014; 58: 1219-1226.

[31] Golas, M., Netsvyetayeke, I., Sikora, M. and Swoboda- Kopec, E. Trends in Antifungal susceptibility of Candidaspecies.Polish Journal of Microbiology.2014;63: 217-222.

[32] Philippe, E., Yok-Ai Que, Jean-Pierre Revelly and Jean-Luc Pagani.Preventing invasive Candidainfections.Journal of hospital infection.2015;89: 302-308.

[33] Badiee, P. and Alborzi, A. (2011). Susceptibility of clinical Candida species isolates to antifungal agents by E-test Iran. Iranian Journal of Microbiology.2011;3: 183-188.

[34] Spellberg, B. J., Filter, S. G. and Edwards, J. E. Current treatment strategies for disseminated candidiasis. Journal of Antimicrobial agents and Chemotherapy.2006; 42: 244-251.

[35] Arendrup, M. C. Epidemiology of invasive candidiasis. Journal of Critical Care.2010;16: 445-452.

[36] Karina, B., Angela, S., Anderson, M. and Arnaldo, L. Genetic diversity of medically important and emerging Candida species causing invasive infection.Journal of BioMedical Central of Infectious Diseases.2015;15: 57.

[37] Guinea, J., Oscar, Z., Pilar, E., Estrella, M., Javier, P., Ferran, S. and Manuel, C. Molecular identification and antifungal susceptibility of yeast isolates causing fungemia. Journal of Antimicrobial Agents and Chemotherapy.2014;58: 1529-1537.

[38] Gregory, A., Jose, A., Williams, A., Kenneth, P. and Clancy, J. Real -World Experience with echinocandin MICs against Candida species. Journal of Antimicrobial Agents and Chemotherapy.2014; 58: 1897-1906.

[39] Sudha, C., Ping, R., Xiaojiang, L. and Tanya, V. Mycology proficiency testing program.Wadsworth Center.2013; 12: 1-5.

[40] Pedro, M., Carole, P. M. and Charlene, W. J. Multi-drug resistant oral Candida species isolated from HIV- positive patients in South Africa and Cameroon. Journal of Diagnostic Microbiology and infectious Disease.2014;79: 222-227. 


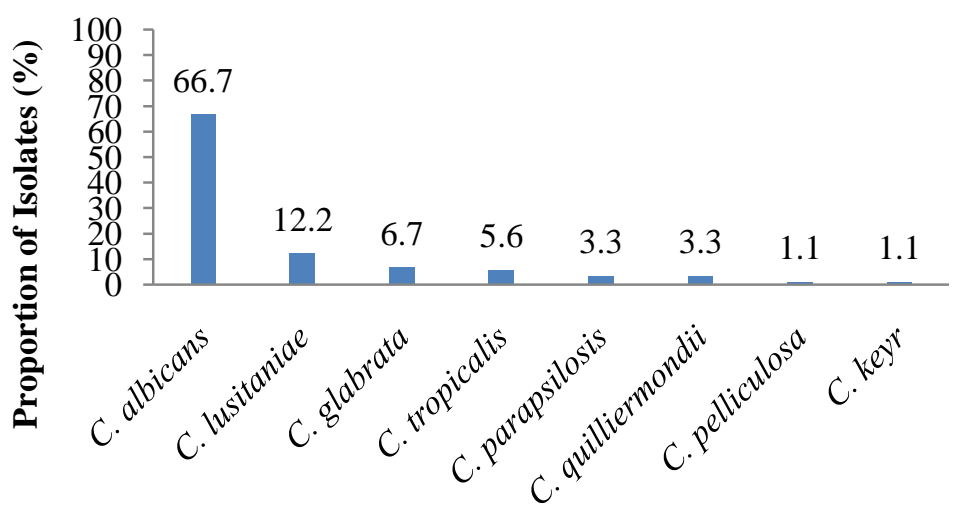

Figure 1: Diversity of Candida species isolated from different clinical specimens

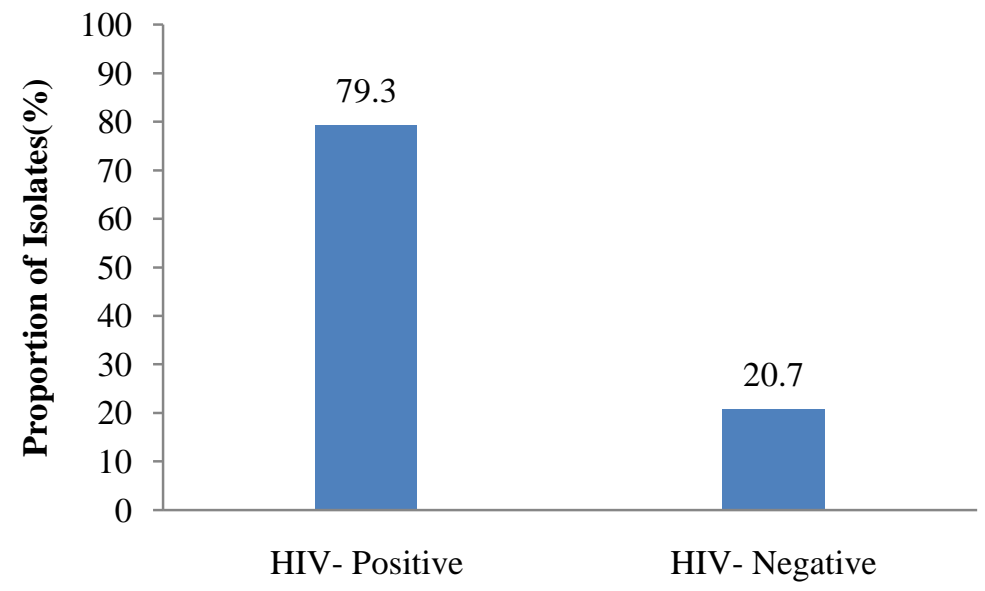

Figure 2: Comparative distribution of resistant Candida species from HIV-negative and HIV-positive patients
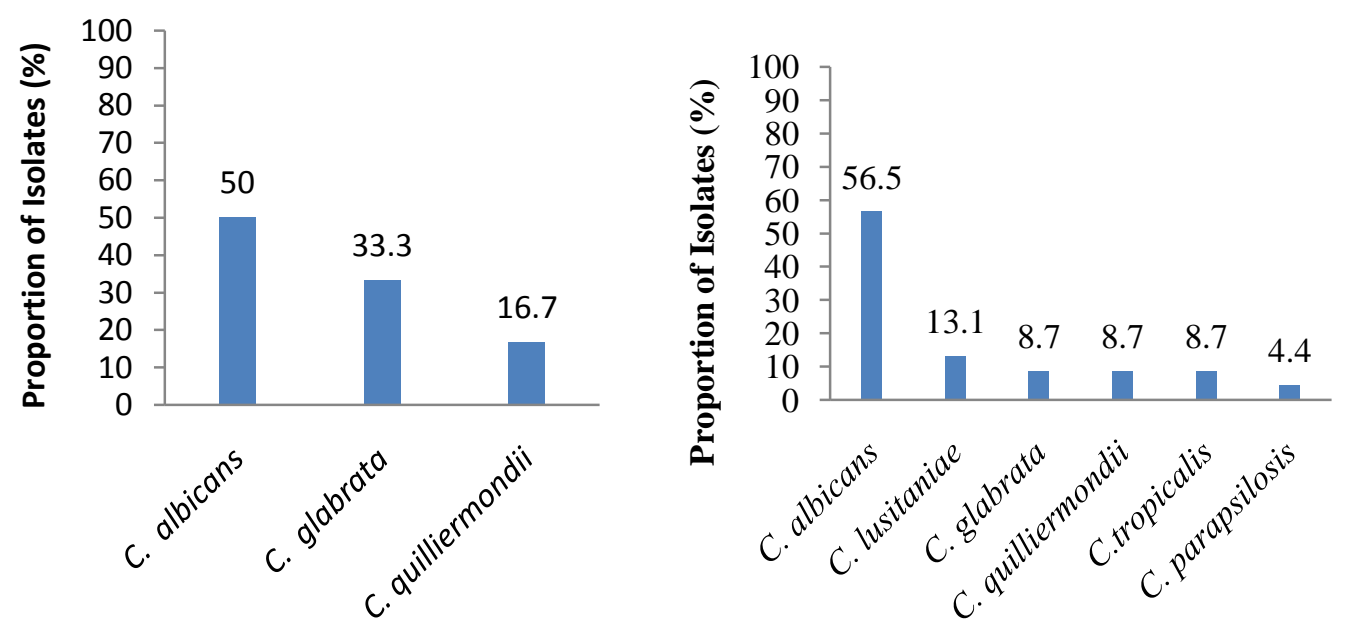

Figure 3: Distribution of drug-resistant Candidaspecies from A) HIV-positive and B) HIV-negative patients

Table 1: Distribution of Candida species by specimens

\begin{tabular}{|l|c|c|c|c|c|}
\hline & \multicolumn{2}{|c|}{ Types of specimen } & & & \\
\hline Species & Urine(\%) & Sputum(\%) & HVS(\%) & Blood(\%) & Total \% \\
\hline C. albicans & $22(59.5)$ & $18(85.7)$ & $18(90)$ & $2(16.7)$ & $60(66.7)$ \\
\hline
\end{tabular}


Distribution and Antifungal Susceptibility ofCandida Species Isolated from Clinical Specimens at the

\begin{tabular}{|l|c|c|c|c|c|}
\hline C. lusitaniae & $4(10.8)$ & $0(0)$ & $0(0)$ & $7(58.3)$ & $11(12.2)$ \\
\hline C. glabrata & $3(8.1)$ & $1(4.8)$ & $2(10)$ & $0(0)$ & $6(6.7)$ \\
\hline C. tropicalis & $2(5.4)$ & $1(4.8)$ & $0(0)$ & $2(16.7)$ & $5(5.6)$ \\
\hline C. parapsilosis & $3(8.1)$ & $0(0)$ & $0(0)$ & $0(0)$ & $3(3.3)$ \\
\hline C. quilliermondii & $2(5.4)$ & $1(4.8)$ & $0(0)$ & $0(0)$ & $3(3.3)$ \\
\hline C. pelliculosa & $0(0)$ & $0(0)$ & $0(0)$ & $1(8.3)$ & $1(1.1)$ \\
\hline C. keyr & $1(2.7)$ & $0(0)$ & $0(0)$ & $0(0)$ & $1(1.1)$ \\
\hline Total \% & $37(41.1)$ & $21(23.3)$ & 20 & $12(13.3)$ & $90 / 96(93.7)$ \\
& & & $(22.2)$ & & \\
\hline
\end{tabular}

Table 2: Concordance between genotypic and phenotypic identification methods for Candida species and other fungal species

\begin{tabular}{|l|c|c|c|}
\hline Organism identified & Genotypic (n) & Phenotypic (n) & Misidentified \\
\hline C. albicans & 60 & 58 & 2 \\
\hline C. lusitaniae & 11 & 11 & 0 \\
\hline C. glabrata & 6 & 6 & 0 \\
\hline C. tropicalis & 5 & 4 & 1 \\
\hline C. parapsilosis & 3 & 4 & 1 \\
\hline C. quilliermondii & 3 & 3 & 0 \\
\hline C. pelliculosa & 1 & 1 & 0 \\
\hline C. keyr & 1 & 1 & 0 \\
\hline C. famata & 0 & 1 & 1 \\
\hline C. krusei & 0 & 1 & 1 \\
\hline Cryptococcus neoformans & 1 & 1 & 0 \\
\hline Saccharomyces cerevisae & 1 & 1 & 0 \\
\hline Cyberlindnerafabianii & 2 & ND & NA \\
\hline Pichia kudrianvzevii & 1 & ND & NA \\
\hline Trichosporonjaponicum & 1 & ND & NA \\
\hline
\end{tabular}

Abbreviation: ND, Not detected: NA, Not applicable.

Table 3: In vitro antifungal susceptibility by MIC $(\mu \mathrm{g} / \mathrm{ml})$ using E - test method.

\begin{tabular}{lllllll} 
Species (no.of isolates) & \multicolumn{1}{c}{ Antifungal agents } & Range & MIC $_{50}$ & MIC $_{90}$ No. of resistant isolates (\%) \\
\hline \multirow{2}{*}{ C. albicans (60) } & Fluconazole & $0.5-256$ & 2.0 & 256 & $11(18.3 \%)$ \\
& Amphotericin B & $0.5-32$ & 1.0 & 1.5 & $6(10 \%)$ \\
& Flucytosine & $0.004-32$ & 0.016 & 0.25 & $2(8.3 \%)$ \\
& Caspofungin & $0.125-32$ & 0.25 & 0.75 & $2(3.3 \%)$ \\
C. lusitaniae (09) & Fluconazole & $0.75-96$ & 1.0 & 96 & $2(22.2 \%)$ \\
& Amphotericin B & $0.75-3$ & 1.0 & 1.5 & $2(22.2 \%)$ \\
& Flucytosine & $0.003-32$ & 0.004 & 6.0 & $1(11.1 \%)$ \\
C. glabrata (06) & Caspofungin & $0.25-1$ & 0.25 & 1 & 0.00 \\
& Fluconazole & $1-16$ & 6 & 16 & 0.00 \\
& Amphotericin B & $1-2$ & 1.5 & 2 & $4(66.6 \%)$ \\
& Flucytosine & $0.002-0.004$ & 0.004 & 0.004 & 0.00 \\
C. tropicalis (05) & Caspofungin & $0.19-0.5$ & 0.25 & 0.5 & 0.00 \\
& & & & & & $1(20 \%)$ \\
& Fluconazole & $1-256$ & 1 & 256 & $1(20 \%)$ \\
& Amphotericin B & $0.75-2$ & 1 & 2 & 0.00 \\
& Flucytosine & $0.006-0.016$ & 0.016 & 0.016 & 0.00
\end{tabular}

Resistance is defined as the following MIC in microgram per millilitre: Flu $\geq 64$; AMB $>1.0 ; \mathrm{FC} \geq 32$; CAS $\geq 32$ using interpretive breakpoint criteria of EUCAST and CLSI (EUCAST 6.1, 2013; MIC test strip, 2014). $\mathrm{MIC}_{50}$ and $\mathrm{MIC}_{90}$ - MIC value to inhibit $50 \%$ and $90 \%$ of the strains tested, respectively.

Table 3: In vitro antifungal susceptibility by MIC $(\mu \mathrm{g} / \mathrm{ml})$ using E - test method (continued). 


\begin{tabular}{|c|c|c|c|c|c|}
\hline Species (no. isolates) & Antifungal agents & Range & $\mathrm{MIC}_{50}$ & $\mathrm{MIC}_{90}$ & No. of resistant isolates ( $\%$ \\
\hline \multirow[t]{4}{*}{ C. parapsilosis (03) } & Fluconazole & $1-8$ & 2 & 8 & 0.00 \\
\hline & Amphotericin B & $0.75-1$ & 0.75 & 1 & 0.00 \\
\hline & Flucytosine & $0.006-32$ & 0.016 & 32 & $1(33.3 \%)$ \\
\hline & Caspofungin & $0.125-0.5$ & 0.25 & 0.5 & 0.00 \\
\hline \multirow[t]{4}{*}{ C. quilliermondii (03) } & Fluconazole & $2-256$ & 256 & 256 & $1(33.3 \%)$ \\
\hline & Amphotericin B & $1-32$ & 0.75 & 32 & $1(33.3 \%)$ \\
\hline & Flucytosine & $0.004-32$ & 0.016 & 32 & $1(33.3 \%)$ \\
\hline & Caspofungin & $0.25-32$ & 0.75 & 32 & $1(33.3 \%)$ \\
\hline \multirow[t]{4}{*}{ C. pelliculosa $(01)$} & Fluconazole & 3 & - & - & 0.00 \\
\hline & Amphotericin B & 1 & - & - & 0.00 \\
\hline & Flucytosine & 0.016 & - & - & 0.00 \\
\hline & Caspofungin & 0.5 & - & - & 0.00 \\
\hline \multirow[t]{4}{*}{ C. kefyr (01) } & Fluconazole & 8 & - & - & 0.00 \\
\hline & Amphotericin B & 1 & - & - & 0.00 \\
\hline & Flucytosine & 0.003 & - & - & 0.00 \\
\hline & Caspofungin & 1.5 & - & - & 0.00 \\
\hline
\end{tabular}

Resistance is defined as the following MIC in microgram per millilitre: Flu $\geq 64$; AMB $>1.0$; FC $\geq 32$; CAS $\geq 32$ using interpretive breakpoint criteria of EUCAST and CLSI (EUCAST 6.1, 2013; MIC test strip, 2014). $\mathrm{MIC}_{50}$ and $\mathrm{MIC}_{90}-\mathrm{MIC}$ value to inhibit $50 \%$ and $90 \%$ of the strains tested respectively.

Table 4: Mean MIC in $\mu \mathrm{g} / \mathrm{ml}$ of antifungal agents on Candida species isolated from HIV-positive and HIVnegative Study participants $(n=96)$.

\begin{tabular}{|c|c|c|c|c|}
\hline \multicolumn{2}{|c|}{ Mean MIC (No. of isolates) } & \multirow[b]{2}{*}{ HIV - $(n=41)$} & \multirow[b]{2}{*}{$95 \% \mathrm{CI}$} & \multirow[b]{2}{*}{$p$ - value } \\
\hline Variables & HIV + (n = 55) & & & \\
\hline Fluconazole & $52.6 \pm 97.6$ & $27.9 \pm 76.7$ & $24.5-60.8$ & 0.17 \\
\hline Amphotericin B & $1.66 \pm 4.2$ & $2.39 \pm 6.8$ & $0.87-3.08$ & 0.51 \\
\hline Flucytosine & $3.73 \pm 10.3$ & $1.58 \pm 6.8$ & $1.01-4.61$ & 0.24 \\
\hline Caspofungin & $2.11 \pm 7.26$ & $1.97 \pm 6.9$ & $0.62-3.49$ & 0.92 \\
\hline
\end{tabular}

Table 5: Antifungal multi-drug resistance pattern of Candida isolates $(\mathrm{n}=5)$
Species
Multi-resistance Pattern
C. albicans
C. guilliermondii
C. lusitaniae
$\mathrm{FLU}+\mathrm{AMB}+\mathrm{FC}+\mathrm{CAS}$
Proportion of Isolation \% (n)
$\mathrm{FLU}+\mathrm{AMB}+\mathrm{FC}+\mathrm{CAS}$
$40(2)$
$20(1)$
$\mathrm{FLU}+\mathrm{AMB} \quad 20(1)$
C. albicans
$\mathrm{AMB}+\mathrm{FC}$
$20(1)$

Abbreviation: FLU, Fluconazole; AMB, Amphotericin B; FC, Flucytosine; CAS, Caspofungin. 